VISUAL EXPERIMENT

\title{
Breeding of zebrafish in the laboratory environment for research development
}

\author{
Sang Ho Kim¹, Chanchal Sharma ${ }^{2}$, Imran Khan² and Sun Chul Kang² \\ Department of ${ }^{1}$ Biology Education and ${ }^{2}$ Biotechnology, Daegu University, Kyoungsan, Kyoung-book 712-714, \\ Republic of Korea.
}

Correspondence to Sun Chul Kang at sckang@daegu.ac.kr

Published: 30 November, 2017

DOI: $10.3329 /$ bjp.v12i4.34143

\section{ABSTRACT}

The zebrafish (Danio rerio) has turn out to be an excellent vertebrate organism for analyzing developmental stages, disease modeling, and screening of drug toxicities due to their minuscule, high fecundity, optical transparency and there close parallels with the genome of human beings. Although maintenance of zebrafish is relatively easy, yet it is a complex process, greatly influenced by various factors including mating behavior, day-light cycle, age, and size of fish. This visual experiment will provide an overview of zebrafish care and maintenance in the laboratory environment. Additionally, the presentation will include information essential for improving the spawning habitat by recreating breeding settings in the lab. Collectively, this experimental memorandum of zebrafish environment can serve as a mean to understand the reproducibility, efficiency of use and its welfare for future research development.

\section{INTRODUCTION}

Understanding all aspects of husbandry is crucial to the success for improving the laboratory animals to produce good scientific results. Over the decades, the zebrafish (Danio rerio) are making a huge breakthrough in biomedical research (Grunwald and Eisen, 2002). The many strengths of zebrafish include their high fecundity, rapid development, small size and external development of embryos (Doke and Dhawale, 2015; Lin et al., 2016). Zebrafish lays enormous eggs that develop externally, allowing the researchers as alternate animal models to perform genetic manipulations and other phenotypic changes in complex organisms. Willian and Poole, (2012) stressed that zebrafish significantly shares $71 \%$ of genetic functions as wells signaling cascade regulatory mechanism similarities with humans. Recently, Howe et al (2017) reported based on OMIM (Online Mendelian Inheritance in Man) tool that $82 \%$ of the genes involved in human disorders bear a strong resemblance with zebrafish. Zebrafish research is also guiding us to understanding the fundamental aspects of developmental stages (Van Slyke et al., 2014), embryogenesis (Veldman and Lin, 2008), fertility (Hoo et al., 2016) and treating human disease (Santoriello and Zon, 2012). Another attribute of zebrafish are their virtual transparency, which allows the observation of developmental processes using non-invasive imaging and tracking of protein/cell markers in biological and disease conditions (Spitsbergen, 2007, Blackburn et al., 2011). Since, zebrafish share much of their genome and signaling cascades with humans which assisted several researchers for understanding the possible mechanism of neurological disorders such as schizophrenia, Huntington's, Parkinson's, and Alzheimer's diseases (Martin-Jimenez, 2015). These advantages have also proved zebrafish to be superior for use in cancer model including carcinogenic exposure and transgenic regulation. The xenograft tumor model in zebrafish provides us on our way to understanding the mechanism between tumor cells and host response (Zhao et al., 2015).

Proper husbandry is essential to the success of experiments performed in zebrafish (Lawrence, 2011). Many researchers have published the widespread use of zebrafish in research laboratories, associated with the considerable low costs, but their culturing facilities requires a very strong knowledge of husbandry to ensure apt maintenance of zebrafish health status(Watts et al., 2012). Keeping this in mind, in this visual experiment, we have described some aspects of zebrafish housing, feeding and breeding in the laboratory environment and tips on factors influencing spawning behavior which can be maintained at very low cost. 
Further, we describe the glimpse into how breeding strategies commonly applied in laboratory settings which can ultimately make an effective scientific research for future successful biological experiments.

\section{MATERIALS AND EQUIPMENTS}

\section{Major equipment}

Zebrafish (Danio rerio) wild type, glass beaker $3000 \mathrm{~mL}(150 \times 215 \mathrm{~mm})$, petri dishes, surgical gloves, laminar flow hood, submersible aquarium heater $\left(20-34^{\circ} \mathrm{C}, 55 \mathrm{~W}\right)$, aerator pump, steel mesh, aquarium plants (e.g. Limnobium laevigatum), light microscope and pipette.

\section{Reagents}

Calcium nitrate, magnesium sulfate, sodium chloride, potassium chloride, HEPES and methylene blue were purchased from the Sigma-Aldrich (USA).

\section{Preparation of reagents}

Embryo medium (E3): The collected embryos were maintained at $28^{\circ} \mathrm{C}$ in embryo medium $[0.2 \mathrm{mM}$ $\mathrm{Ca}\left(\mathrm{NO}_{3}\right)_{2}, 0.13 \mathrm{mM} \mathrm{MgSO}_{4}, 19.3 \mathrm{mM} \mathrm{NaCl}, 0.23 \mathrm{mM} \mathrm{KCl}, 0.1 \%$ methylene blue and $1.67 \mathrm{mM}$ HEPES, equilibrated to $\mathrm{pH} 7.0]$.

\section{VIDEO CLIP}

Duration: 2 min $51 \mathrm{sec}$

\section{PROTOCOL}

\section{Maintenance of zebrafish}

1. Zebrafish were obtained from the existing breeding stock at the local aquarium in Daegu, South Korea, and kept in a proper aerating system to maintain the oxygen and water quality required for a healthy environment. Though different sized breeding tanks were available we utilized 3L glass beaker in our laboratory for economic purpose.

2. The temperature of tank system was generally maintained at $28 \pm 0.5^{\circ} \mathrm{C}$ using the submersible heater $(55 \mathrm{~W})$ and the lighting condition are 14:10 hours (Westerfield, 2007).

3. Water was dechlorinated either by reverse osmosis purification system or it can be dechlorinated by ageing the water for at least 48 hours.

4. Ideally, the $\mathrm{pH}$ of the water should be 7.0 to 8.0 in the tank when necessary, adjusting $\mathrm{pH}$ with sodium bicarbonate. It is recommended to remove and add $10 \%$ of the dechlorinated water daily.

5. Next step is to disinfect and clean the tank by using laboratory disinfectant ( $10 \%$ sodium hypochlorite) in order to avoid algal growth and fish waste accumulation. Once the tanks have been cleaned, fill the tanks with fresh water. This step is highly recommended because zebrafish will not breed if the water conditions are not good.

\section{Feeding of zebrafish}

1. In our laboratory, we fed zebrafish twice a day with commercially available diet (Tabia-Top Meal, South Korea).

2. We used the size from $300 / 400$ microns for adult zebrafish and 100 microns for larval feeding.

3. To avoid food intake probability in zebrafish, we are suggesting to turning off the aeration system before feeding.

\section{Breeding conditions}

1. Male and female zebrafish recognition is the initial step for successful breeding. Usually, females can be easily distinguished from males because of their bigger underbelly (Schilling, 2002).

2. Zebrafish initiate breeding at the onset of white light. Fertilized eggs can be obtained either through intank breeding or pairwise breeding. In our laboratory, we preferred pairwise breeding for regular embryo collection.

3. Pairwise breeding is usually initiated after feeding. After sex determination, male and female were kept in 
a separate tank under optimum conditions for at least $8 \mathrm{~h}$ or until the night before breeding.

4. We assembled two breeding tanks set-up in our lab (1) simple meshed tank and (2) pebbles and aquatic plants filled the tank for analyzing better breeding efficacy.

5. We transferred one male and two female zebrafish to two breeding tanks and left undisturbed overnight at dark.

6. Next morning shortly after the onset of white light, allow the spawning to occur undisturbed for $30 \mathrm{~min}$ to 1 hour or notice the visibility of sufficient embryos is laid down at the bottom of the tank.

7. Return the fishes to their tanks and collect the embryo by pipette suction or by a strainer, wash the embryos thrice with tap water and staged according to Kimmel et al, (1995).

8. Finally, the total number of eggs produced from both breeding tanks were compared and illustrated in visual section. Eggs free of macroscopically discernable symptoms of infection and disease were picked and maintained in embryo medium at $28 \pm 0.5^{\circ} \mathrm{C}$ until experiments.

\section{DISCUSSION}

Growing evidence on zebrafish research has provided its place as an excellent animal model over other laboratory animals due to its genetic resemblance to human, cost-effective and transparent embryo (Spence et al., 2008). This attributes greatly witnessed its importance in behavioral research (Blaser et al., 2010), genetics (Gerlai, 2003) and drug screening (Eddins et al., 2009). Despite of its wide range of growing prevalence in research program, there are several challenges that arise during their maintenance under sub-optimal conditions (Avdesh et al., 2012). Hence, complete understanding of the zebrafish husbandry is essential for the successful outcome of laboratory experiments. In this video, we emphasize an outline protocol for zebrafish maintenance, feeding, breeding and collection of eggs based on our laboratory experiences.

\section{Housing and maintenance}

Water qualities are the pervasive factors determining the physiochemical properties as well as healthy environment for zebrafish management. It is crucial to provide them with a hygienic condition in a wellfunctioning aeration system. Therefore, the quality of water such as alkalinity, $\mathrm{pH}$, temperature, hardness, ammonia, dissolved oxygen and salinity was checked on a regular basis. Under sub-optimal conditions, holding zebrafish in tap water is not recommended because of chlorine, and other potential contaminants (Kent et al., 2014). In order to make tap water safe from contaminants, we therefore passed water through a purification system (reverse osmosis unit) and replaced about $10 \%$ of fresh water daily in the breeding tank. Water can also be dechlorinated by ageing for at least 48hr. We favors Westerfield (2007) recommended concentrations and dosages of chlorine $(25-50 \mathrm{ppm})$ and nitrate $(<50 \mathrm{mg} / \mathrm{L})$ contents in the water system as these contaminants significantly reduces body salts and energy levels in zebrafish.

\section{Temperature and feeding}

Temperature control in sub-optimal settings is also exceptionally important because of its profound effects on biochemical functions. As a eurythermal species, zebrafish can tolerate up to 6.7 to $41.7^{\circ} \mathrm{C}$ but optimum temperature has not formally defined yet (Schaefer and Ryan, 2006). We maintained the temperature of near $28^{\circ} \mathrm{C}$, or $80^{\circ} \mathrm{F}$ which is universally accepted for zebrafish culture. Likewise, to retain the circadian rhythm, lights are controlled on a cycle with 14 hours of light and 10 hours of darkness.

Habitually, zebrafish consumes zooplankton and insects, which has been replaced by commercial diet during the present investigation For consideration, never be overfed zebrafish as this may increase toxic nitrite level in the water which can ultimately affects viability and breeding efficiency (Westerfield, 2007). We recommend feeding 2-3 times per day, while feeding air pump and/or water circulating systems should be turn off which allows the fish to eat the complete food for $10 \mathrm{~min}$.

\section{Breeding and Collection of eggs}

Zebrafish fertility conditions usually begin at 3rd month and start to decline after the 18 months of age. Accumulating evidence on understanding the reproductive biology of fish has fueled its applications in breeding techniques (Adatto et al., 2011; Nasiadka and Clark, 2012). There has been generally little examination concerning the common environmental inclinations of the zebrafish and there is plausibility of sub-optimal husbandry conditions. Therefore, examination of zebrafish reproduction in the laboratory setting is of high importance for husbandry. During the initial phase of study, we experienced problems with breeding conditions, however using female and male zebrafish (2:1 ratio) along with altered nature like settings (e.g. mesh, pebbles and aquatic plants) in breeding tank helped solve the problem. Moreover, feeding with commercial diet two-three times a day under optimum temperature $\left(28^{\circ} \mathrm{C}\right)$ further improved 
breeding efficacy. Next, we made comparative analysis of egg numbers in two breeding tank set-up one holding simple steel mesh and another tank containing pebbles and aquatic plants (Limnobium laevigatum). After spawning, we noticed that when compared to simple meshed tank, tank containing natural like environment significantly improved the no of egg production. The eggs without any sign of abnormalities were evaluated under light microscope and then transferred in to embryo medium until experiments. Therefore, we suggest that maintaining nature like environment always improving the breeding in fish also keeping a record of the number of embryos laid by fish from each tank is also recommended.

\section{CONCLUSION}

In conclusion, the application of the zebrafish moves beyond developmental genetics to the modeling of human disease, drug discovery, toxicology, and other diverse fields, it is becoming increasingly apparent that there is a need to develop scientifically based standards for husbandry, management, and care of the fish. Indeed, the fact that the zebrafish is relatively easy to rear and propagate in the laboratory is a major, but often overlooked, contributing factor in its rise to prominence. For the same reason, the science of husbandry and zebrafish aquaculture has remained undeveloped. Collectively, the video on zebrafish maintenance and husbandry in the laboratory condition will provide an understanding of breeding management strategies that can influence the experimental landscape of the fish.

\section{REFERENCES}

Adatto I, Lawrence C, Thompson M, Zon LI. A new system for the rapid collection of large numbers of developmentally staged zebrafish embryos. PLoS One. 2011; 6: e21715.

Avdesh A, Chen M, Martin-Iverson MT, Mondal A, Ong D, Rainey-Smith S, Taddei K, Lardelli M, Groth DM, Verdile G, Martins RN. Regular care and maintenance of a zebrafish (Danio rerio) laboratory: An introduction. J Vis Exp. 2012: 18: 4196.

Blackburn JS, Liu S, Raimondi AR, Ignatius MS, Salthouse CD, Langenau DM. High throughput imaging of adult fluorescent zebrafish with an LED fluorescence macroscope. Nat Protoc. 2011; 6: 229-41.

Blaser RE, Chadwick L, McGinnis GC. Behavioral measures of anxiety in zebrafish (Danio rerio). Behav Brain Res. 2010; 208: 56-62.

Doke SK, Dhawale SC. Alternatives to animal testing: A review. Saudi Pharm J. 2015; 23: 223-29.

Eddins D, Petro A, Williams P, Cerutti DT, Levin ED. Nicotine effects on learning in zebrafish: The role of dopaminergic systems. Psychopharmacology (Berl) 2009; 202: 103-09.

Gerlai R. Zebrafish: An uncharted behavior genetic model. Behav Genet. 2003; 33: 461-68.

Grunwald DJ, Eisen JS. Headwaters of the zebrafish: Emergence of a new model vertebrate. Nat Rev Genet. 2002; 3: 71724.

Hoo JY, Kumari Y, Shaikh MF, Hue SM, Goh BH. Zebrafish: A versatile animal model for fertility research. Biomed Res Int. 2016; 2016: 9732780

Howe DG, Bradford YM, Eagle A, Fashena D, Frazer K, Kalita P, Mani P, Martin R, Moxon ST, Paddock H, Pich C, Ramachandran S, Ruzicka L, Schaper K, Shao X, Singer A, Toro S, Van Slyke C, Westerfield M. The zebrafish model organism database: New support for human disease models, mutation details, gene expression phenotypes and searching. Nucleic Acids Res. 2017; 45: D758-68.

Kent ML, Buchner C, Barton C, Tanguay RL. Toxicity of chlorine to zebrafish embryos. Dis Aquat Organ. 2014; 107: 23540 .

Kimmel CB, Ballard WW, Kimmel SR, Ullmann B, Schilling TF. Stages of embryonic development of the zebrafish. Dev Dyn. 1995; 203: 253-310.

Lawrence C. Advances in zebrafish husbandry and management. Methods Cell Biol. 2011; 104: 429-51.

Lin CY, Chiang CY, Tsai HJ. Zebrafish and Medaka: New model organisms for modern biomedical research. J Biomed Sci. 2016; 23: 19.

Martin-Jimenez R1, Campanella M, Russell C. New zebrafish models of neurodegeneration. Curr Neurol Neurosci Rep. 2015; 15: 33.

Nasiadka A, Clark MD. Zebrafish breeding in the laboratory environment. ILAR J. 2012; 53: 161-68.

Santoriello C, Zon LI. Hooked! Modeling human disease in zebrafish. J Clin Invest. 2012; 122: $2337-43$.

Schaefer J, Ryan A. Developmental plasticity in the thermal tolerance of zebrafish Danio rerio. J Fish Biol. 2006; 69: 722734. 
Schilling TF. Zebrafish: A practical approach. New York, Oxford University Press, 2002.

Spence R, Gerlach G, Lawrence C, Smith C.The behaviour and ecology of the zebrafish, Danio rerio. Biol Rev Camb Philos Soc. 2008; 83: 13-34.

Spitsbergen J. Imaging neoplasia in zebrafish. Nat Methods.2007; 4: 548-49.

Van Slyke CE, Bradford YM, Westerfield M, Haendel MA. The zebrafish anatomy and stage ontologies: Representing the anatomy and development of Danio rerio. J Biomed Semantics. 2014; 5: 12.

Veldman MB, Lin S. Zebrafish as a developmental model organism for pediatric research. Pediatr Res. 2008; 64: 470-76.

Watts SA, Powell M, D'Abramo LR. Fundamental approaches to the study of zebrafish nutrition. ILAR J. 2012; 53: 14460 .

Westerfield M. The zebrafish book: A guide for the laboratory use of zebrafish (Danio rerio). 5th ed. Oregon, University of Oregon Press, 2007.

Williams CM, Poole AW. Using zebrafish (Danio rerio) to assess gene function in thrombus formation. Methods Mol Biol. 2012; 788: 305-19.

Zhao S, Huang J, Ye J. A fresh look at zebrafish from the perspective of cancer research. J Exp Clin Cancer Res. 2015; 34: 80.

\section{PRECAUTION}

Points should be considered before initiating breeding proficiency

- Usually, algae and solid wastes can layering up in the tank and net, we recommended that the tank, mesh and/or net must be cleaned with $70 \%$ ethanol, neutralize in water and allowed to dry before being re-used or else which is otherwise toxic to fish.

- Pair wise breeding should not be performed for more than two days, it will reduce mating efficiency.

- To enhance breeding cycle of fish, breeding should be undertaken at regular basis even if eggs are not required.

- It is recommended to allow more female than male in breeding tanks because male fish changes their mating partners on a daily basis.

- After 5 day post-fertilization, young larvae can be fed with commercial food of $\sim 100$ microns in size and size can be slowly increased to $>100$ microns.

\section{ACKNOWLEDGEMENT}

This research was supported by a Daegu University Research Grant from the Republic of Korea, 2014.

\section{CONFLICT OF INTEREST}

The authors declare that they have no competing interests. 